\title{
An Efficient Discrete Model of a Simple Biped with a Torso
}

\author{
$\underline{\text { Sarra A. Gismelseed }}{ }^{1}$, Amur S. Al Yahmedi ${ }^{2}$, Riadh Zaier $^{2}$, Hassen M. Ouakad ${ }^{2}$, Issam \\ Bahadur $^{2}$ \\ ${ }^{1}$ Department of Mechanical \& Industrial \\ Engineering \\ Sultan Qaboos University \\ Al-Khoud, Muscat, 123, Oman \\ sarraabbasher@gmail.com \\ ${ }^{2}$ Department of Mechanical \& Industrial \\ Engineering \\ Sultan Qaboos University \\ Al-Khoud, Muscat, 123, Oman \\ [amery, zaier, houakad, \\ bahdoor@squ.edu.om]
}

\begin{abstract}
Human locomotion involves a complex integration of muscles activity, central nervous system, and sensory information. Attempts to describe and understand the biomechanics of human locomotion have been made experimentally and mathematically (simulation). Mathematically, biped models with various complexities have been used to study human locomotion using different numerical methods and many features of human locomotion have been verified. In this paper, an optimization based prediction of human gait with its essential features using simple biped with torso is formulated. The biped can "qualitatively" mimic many features of human locomotion including the general behavior of human gait during running and walking.
\end{abstract}

Keywords: Discrete Mechanics, Optimization, Gait, Biped, Torso.

\section{INTRODUCTION}

Many researchers have studied human gait by modeling human body as a simple mechanical system represented mathematically as biped models that can describe the basics of human motion. Many studies in the literature on humanoid robots have focused in deriving the model using standard continuous-time mechanics. In [1], a gait trajectory of biped model in continuous time domain has been provided using simple technique that is based on the symmetric features in the dynamics of this compass-type model. The motion obtained by their technique resembles the phenomenon of a passive dynamic walking, since the motion contains swing phase and a foot collision taking place one after another. Srinivasan et. al., [2] have simulated human gait using an inverted pendulum model with the assumption of a rigid human body and massless legs. The model in [2] generated the energy-based optimal gait of three distinct types of human gait that are; i) walking, ii) inverted pendulum run, and iii) running. The discrete mechanics, on the other hand, have been recently applied to derive biped models. Compass-type biped model and discrete mechanics are used in [3] to formulate a gait generation problem. They have verified the generation of a stable gait by formulating a constrained nonlinear optimization problem in which the model minimizes the angular velocities using both impact and swing phases models. In 2015, Sun et. al. [4] have studied periodic gait optimization problem of the bipedal walking robot using discrete mechanics. The optimization problem was numerically solved using a class of global and feasible sequential quadratic programming algorithms [5][6]. Their study has shown that the algorithm can converge to a stable gait cycle by selecting a proper initial guess of the gait [4].

Biomedical researches have also focused on applying optimization techniques and algorithms to predict various types of human motion and dynamics. For a stable and a converging algorithm, several factors should be considered in the optimization routine such as the surrounded environment reaction forces and system inertia forces. Optimization algorithms can be applied for gait prediction, lifting, pushing, and pulling movements, to name a few. Prediction of these motions requires different optimization formulations and two or more tasks can be combined 
together in a single optimization problem [7].

Numerical simulations are actively used today in different engineering and science fields to simulate the behavior of dynamic systems which was commonly involved to solve continuous models when simulating dynamical systems. However, when long-time simulation is required, it is frequently necessary to focus on preserving the qualitative behavior of the system such as transformation between velocity and momenta. The idea behind preserving energy in discrete mechanics is that, unlike other numerical methods, we discretize the basic principle of the mechanical system not the final differential equations. Therefore, symplectic methods [8] [9] (i.e., methods that are based on energy conservation) are becoming more widely used to simulate systems that have long-time simulation features such as humanoid robots. With these types of systems, it will be difficult to involve non-symplectic methods such as Runge-Kutta to simulate the behavior of those systems because it dissipates energy with time and hence increases the numerical error in the simulation [10]. To overcome the numerical errors with long- time simulation, discrete Lagrangian mechanics is introduced by considering a discrete Hamilton's principle [9][11]. In discrete mechanics, the Euler-Lagrange equations of the nonlinear mechanical systems are discretized. This way of formulating the equations of motion in mechanics has the capability of analysis with great compatibility with computers because of fewer errors in judgment with other similar numerical methods such as Euler method and Runge-Kutta method [7]. Furthermore, simulations can be performed for relatively large sampling times. Due to their symplectic nature, discrete mechanics are perfect for simulations that take long time. Therefore, the obtained discrete trajectories exhibit a good energy performance with time [12].

\section{MATHEMATICAL MODEL}

In this work, the developed model is based on the concept of the inverted pendulum model with the torso in order to generate more realistic information by mimicking human locomotion. The derivation of the model is based on the principle of discrete mechanics since it considers as a symplectic method that preserves momenta associated to symmetries of the system, and it also has perfect long-time energy compared to other numerical methods as discussed previously.

\subsection{Discrete mechanics}

In discrete Lagrangian, a path $q(t)$ for $t \in[0, T]$ is changed to a discrete path $q:\left\{t_{0}, \ldots, t_{k}, \ldots, t_{N}=T\right\}$, where $k \in \mathbb{N}$ and $N \in \mathbb{N} .\left(q_{k}\right.$ is viewed as an estimation of $\left.q\left(t_{k}\right)\right)$. The Lagrangian $L(t)$ if estimated on each interval $\left[t_{k}, t_{k+1}\right]$ by a discrete Lagrangian $L_{d}\left(q_{k}, q_{k+1}, h\right)$; where, $h$ is the time interval between two samples [9]:

$$
h=t_{k+1}-t_{k} .
$$

The formula of discrete Lagrangian is obtained by estimating the integral of the continuous-time Lagrangian over a small interval of time $h$ using the midpoint rule [12].

$$
\begin{gathered}
q=\frac{q_{k+1}+q_{k}}{2}, \\
v=\frac{q_{k+1}-q_{k}}{h}, \\
L_{d}\left(q_{k+1}, q_{k}\right)=\int_{t_{k}}^{t_{k+1}} L(q, v) d t=h L\left(\frac{q_{k+1}+q_{k}}{2}, \frac{q_{k+1}-q_{k}}{h}\right) .
\end{gathered}
$$

Then we can approximate the discrete action $S_{d}$ as a sum of discrete Lagrangian and using Hamiltonian principle: if the path is slightly "varied", action is unchanged to the first order [13].

$$
\delta S_{d}=\sum_{k=0}^{n-1} \delta L_{d}\left(q_{k+1}, q_{k}\right)=0 .
$$

Then, we minimize $S_{d}$ with respect to perturbations of trajectories. Using the boundary conditions for $\delta q$, we now have summation that must vanish for all values of $\delta q$ and one gets 
the discrete Lagrangian formula [9]:

$$
D_{2} L_{d}\left(q_{k-1}, q_{k}\right)+f_{d}{ }^{+}\left(q_{k-1}, q_{k}\right)+D_{1} L_{d}\left(q_{k}, q_{k+1}\right)+f_{d}{ }^{-}\left(q_{k}, q_{k+1}\right)=0 .
$$

Where, $f_{d}{ }^{-}\left(q_{k}, q_{k+1}\right)$ and $f_{d}{ }^{+}\left(q_{k-1}, q_{k}\right)$ left and right discrete forces, respectively, and:

$D_{1} L_{d}\left(q_{k}, q_{k+1}\right)$ : is the first derivative with respect to the first argument of $L_{d}$ (i.e., $q_{k}$ ).

$D_{2} L_{d}\left(q_{k-1}, q_{k}\right)$ : is the first derivative with respect to the second argument of $L_{d}$ (i.e., $q_{k}$ ).

And we have the discrete force related to the continuous force as the follows:

$$
f_{d}{ }^{+}\left(q_{k-1}, q_{k}, t_{k-1}, t_{k}\right)=f_{d}^{-}\left(q_{k}, q_{k+1}, t_{k}, t_{k+1}\right)=\frac{h}{2} f_{c}\left(\frac{q_{k+1}+q_{k}}{2}, \frac{q_{k+1}-q_{k}}{h} \frac{t_{k+1}+t_{k}}{2}\right) \text {. }
$$

\subsection{Biped model}

Mechanical and mathematical biped models have been used to describe human basic motion. Although many studies have concentrated on the lower extremities in their models, there are two reasons that invite one to study the role of the torso in gait. First, most of the body weight is concentrated in the upper part of the body. The torso makes about $70 \%$ of total body weight. Second, the center of gravity of the whole body is in the upper body specifically in the torso, approximately $33 \mathrm{~cm}$ above the hip joint [14].

The proposed model shown in Fig. 1 has two point masses, one for the hip and the other for the rigid torso, and the two legs are considered to be massless. The hip mass $m_{H}$ has a position $\left(x_{H}, y_{H}\right)$ and the torso a position $\left(x_{T}, y_{T}\right)$ at time $t$. The motion of the torso is controlled via a torque $\tau(t)$ applied between the torso and the stance leg. A single telescopic axial actuator is used to combine flexion of the three joints in the leg: hip, knee, and ankle, which consequently cause variations of the leg length $q(t)$. This actuator transmits a compressive time-varying force $F(t)$ and both legs are assumed to have identical profiles of leg length and ground reaction force.

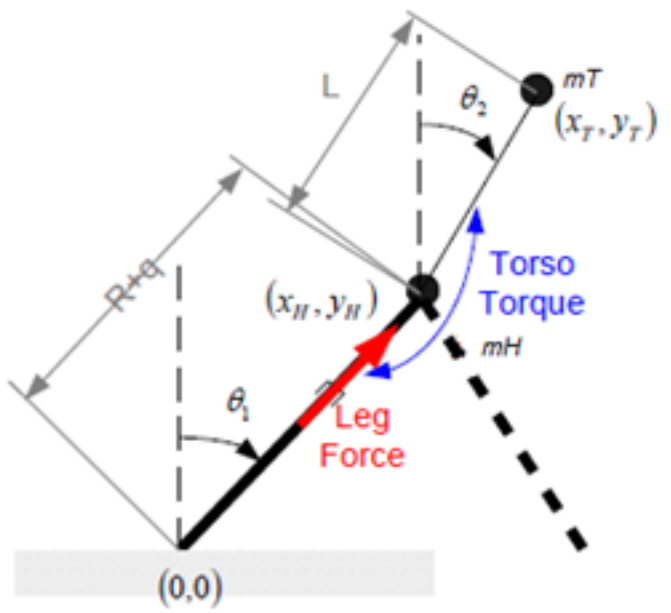

Figure 1. Biped model with a torso

The discrete Euler Lagrange equation of the biped is given by: 


$$
\begin{aligned}
& L_{d}\left(\theta_{1 k}, \theta_{1 k+1}, \theta_{2 k}, \theta_{2 k+1}, q_{k}, q_{k+1}\right)=\frac{1}{2} h\left(m_{H}+m_{T}\right)\left(R+\frac{\Sigma q_{k}}{2}\right)^{2}\left(\frac{\Delta \theta_{1 k}}{h}\right)^{2} \\
& +\frac{1}{2} h\left(m_{H}+m_{T}\right)\left(\frac{\Delta q_{1 k}}{h}\right)^{2}+\frac{1}{2} h m_{T} L^{2}\left(R+\frac{\Sigma q_{k}}{2}\right)^{2}\left(\frac{\Delta \theta_{2 k}}{h}\right)^{2} \\
& +h m_{T} L\left(R+\frac{\Sigma q_{k}}{2}\right)\left(\frac{\Delta \theta_{1 k}}{h}\right)\left(\frac{\Delta \theta_{2 k}}{h}\right) \cos \left(\frac{\Sigma \theta_{1 k}}{2}-\frac{\Sigma \theta_{2 k}}{2}\right) \\
& +h m_{T} L\left(\frac{\Delta \theta_{1 k}}{h}\right)\left(\frac{\Delta q_{k}}{h}\right) \sin \left(\frac{\sum \theta_{1 k}}{2}-\frac{\sum \theta_{2 k}}{2}\right) \\
& -h\left(m_{T}+m_{H}\right) g\left(R+\frac{\sum q_{k}}{2}\right) \cos \left(\frac{\sum \theta_{1 k}}{2}\right)-h m_{T} g L \cos \left(\frac{\sum \theta_{2 k}}{2}\right) .
\end{aligned}
$$

Note: $\Delta \theta_{1 k}=\theta_{1 k+1}-\theta_{1 k}, \sum \theta_{1 k}=\theta_{1 k+1}+\theta_{1 k}, \Delta \theta_{2 k}=\theta_{2 k+1}-\theta_{2 k}, \sum \theta_{2 k}=\theta_{2 k+1}+\theta_{2 k}$, $\Delta q_{k}=q_{k+1}-q_{k}$ and $\sum q_{k}=q_{k+1}+q_{k}$.

The Discrete Euler Lagrange formulation is used to find the equations of the biped by assuming only one-foot contacts the ground.

$$
\begin{aligned}
& D_{2} L_{d}\left(\theta_{1 k-1}, \theta_{1 k}, \theta_{2 k-1}, \theta_{2 k}, q_{k-1}, q_{k}\right)+f_{d}^{+}\left(\theta_{1 k-1}, \theta_{1 k}, \theta_{2 k-1}, \theta_{2 k}, q_{k-1}, q_{k}\right) \\
& +D_{1} L_{d}\left(\theta_{1 k}, \theta_{1 k+1}, \theta_{2 k}, \theta_{2 k+1}, q_{k}, q_{k+1}\right)+f_{d}^{-}\left(\theta_{1 k}, \theta_{1 k+1}, \theta_{2 k}, \theta_{2 k+1}, q_{k}, q_{k+1}\right)=0,
\end{aligned}
$$

Where,

$$
\left.\begin{array}{rl}
D_{1} L_{d}= & {\left[\begin{array}{l}
\frac{\partial L_{d}\left(\theta_{1 k}, \theta_{1 k+1}, \theta_{2 k}, \theta_{2 k+1}, q_{k}, q_{k+1}\right)}{\partial \theta_{1 k}} \\
\frac{\partial L_{d}\left(\theta_{1 k}, \theta_{1 k+1}, \theta_{2 k}, \theta_{2 k+1}, q_{k}, q_{k+1}\right)}{\partial \theta_{2 k}} \\
\frac{\partial L_{d}\left(\theta_{1 k}, \theta_{1 k+1}, \theta_{2 k}, \theta_{2 k+1}, q_{k}, q_{k+1}\right)}{\partial q_{k}}
\end{array}\right],} \\
D_{2} L_{d}= & {\left[\begin{array}{l}
\frac{\partial L_{d}\left(\theta_{1 k-1}, \theta_{1 k}, \theta_{2 k-1}, \theta_{2 k}, q_{k-1}, q_{k}\right)}{\partial \theta_{1 k}} \\
\frac{\partial L_{d}\left(\theta_{1 k-1}, \theta_{1 k}, \theta_{2 k-1}, \theta_{2 k}, q_{k-1}, q_{k}\right)}{\partial \theta_{2 k}}
\end{array}\right] .} \\
\frac{\partial L_{d}\left(\theta_{1 k-1}, \theta_{1 k}, \theta_{2 k-1}, \theta_{2 k}, q_{k-1}, q_{k}\right)}{\partial q_{k}}
\end{array}\right] .
$$

The discrete forces are the Ground Reaction Force (GRF) and the torque acting on the leg and the torso.

$$
f_{d}^{+}\left(\theta_{1 k-1}, \theta_{1 k}, \theta_{2 k-1}, \theta_{2 k}, q_{k-1}, q_{k}\right)=f_{d}^{-}\left(\theta_{1 k}, \theta_{1 k+1}, \theta_{2 k}, \theta_{2 k+1}, q_{k}, q_{k+1}\right)=\frac{h}{2}\left[\begin{array}{c}
\tau \\
-\tau \\
F
\end{array}\right] .
$$

The biped model has the dimensions descried in the following table.

Table 1. Dimensions of biped model with a torso

\begin{tabular}{|c|l|c|}
\hline Symbol & \multicolumn{1}{|c|}{ Description } & Value \\
\hline$m$ & Total body mass $(\mathrm{Kg})$ & 10 \\
\hline$m_{T}$ & Mass of the torso $(\mathrm{Kg})$ & $\frac{2}{3} m$ \\
\hline
\end{tabular}




\begin{tabular}{|c|l|c|}
\hline$m_{H}$ & Mass of the hip (Kg) & $\frac{1}{3} m$ \\
\hline$L$ & Distance from hip to the center of mass of the torso $(\mathrm{m})$ & 0.46 \\
\hline$R$ & Nominal Leg Length $(\mathrm{m})$ & 1 \\
\hline$g$ & Gravitational acceleration $(\mathrm{m} / \mathrm{s})$ & 9.81 \\
\hline
\end{tabular}

\section{OPTIMIZATION AND SIMULATION RESULTS}

During human locomotion, the body consumes energy to perform work. Earlier studies tried to link the metabolic requirements of walking and running to the positive mechanical power required to raise and accelerate the body's center of mass and accelerate the limbs relative to the center of mass in animals and humans [15]. Metabolic cost of transport is the metabolic energy required to move a unit body weight or mass a unit distance. Given a specific step size $d$ and if the biped starts its step with the nominal leg length $R+q(1)=R$; we search for the control approach that gives the minimum cost of transport that is

$$
J=\sum_{k=1}^{N} h \frac{\left|F_{k}(t)\left(\frac{\Delta q_{k}}{h}\right)\right|+\left|\tau_{k}(t)\left(\frac{\Delta \theta_{1 k}}{h}-\frac{\Delta \theta_{2 k}}{h}\right)\right|}{2 m g d} .
$$

Subject to dynamics constraints of the biped and satisfying periodicity constraints of the gait:

- The torso starts and ends the step with the same position and velocity.

$$
\begin{gathered}
\theta_{2}(N+1)=\theta_{2}(1), \\
\frac{\theta_{2}(N+1)-\theta_{2}(N)}{h}=\frac{\theta_{2}(2)-\theta_{2}(1)}{h} .
\end{gathered}
$$

- $\quad$ The hip starts and ends the step with the same velocity in both $\mathrm{X}$ and $\mathrm{Y}$ directions.

$$
\begin{aligned}
&(R+q(N+1)) \cos \left(\theta_{1}(N+1)\right)\left(\frac{\theta_{1}(N+1)-\theta_{1}(N)}{h}\right)+\left(\frac{q(N+1)-q(N)}{h}\right) \sin \left(\theta_{1}(N+1)\right) \\
&=(R+q(1)) \cos \left(\theta_{1}(1)\right)\left(\frac{\theta_{1}(2)-\theta_{1}(1)}{h}\right)+\left(\frac{q(2)-q(1)}{h}\right) \sin \left(\theta_{1}(1)\right), \\
&-(R+q(N+1)) \sin \left(\theta_{1}(N+1)\right)\left(\frac{\theta_{1}(N+1)-\theta_{1}(N)}{h}\right)+\left(\frac{q(N+1)-q(N)}{h}\right) \cos \left(\theta_{1}(N+1)\right) \\
&=(R+q(1)) \sin \left(\theta_{1}(1)\right)\left(\frac{\theta_{1}(2)-\theta_{1}(1)}{h}\right)+\left(\frac{q(2)-q(1)}{h}\right) \cos \left(\theta_{1}(1)\right) .
\end{aligned}
$$

- $\quad$ The hip starts and ends the step with the same position in $\mathrm{Y}$ direction.

$$
(R+q(N+1)) \cos \left(\theta_{1}(N+1)\right)=(R+q(1)) \cos \left(\theta_{1}(1)\right) .
$$

- The difference between the final and initial positions of the hip is the step length $\mathrm{d}$.

$$
d=(R+q(N+1)) \sin \left(\theta_{1}(N+1)\right)-(R+q(1)) \sin \left(\theta_{1}(1)\right) .
$$

In addition to the pervious constraints, we represent the flexion and extension of knee and ankle joints with a maximum variation of $10 \%$ of the nominal length of the leg [16].

$$
1-\operatorname{Max} E x t<q<1+\operatorname{Max} E x t .
$$

Where, MaxExt is the maximum Extension of the length.

The optimization procedure was run with different initial conditions to search for the optimal solutions as we vary the desired step size $d$ and biped speed $v$. For each set of these two parameters, the optimizer converges to a distinctive result (see sections 3.1 and 3.2) that 
describes the optimal gait.

\subsection{Walking}

Humans tend to walk at low speed and the walking gait can be identified from the profile of GRF (Ground Reaction Force) of the gait. Fig. 2 shows the gait pattern for $0.6 \mathrm{~m}$ step size with velocity of $1.566 \mathrm{~m} / \mathrm{s}$. The optimal gait for these parameters, based on the minimum cost of transport, is walking. The profile of normal GRF has two symmetric peaks that represent two phases of gait cycle, the heel strike and push off. These two phases occur at the beginning and the end of gait cycle [17].

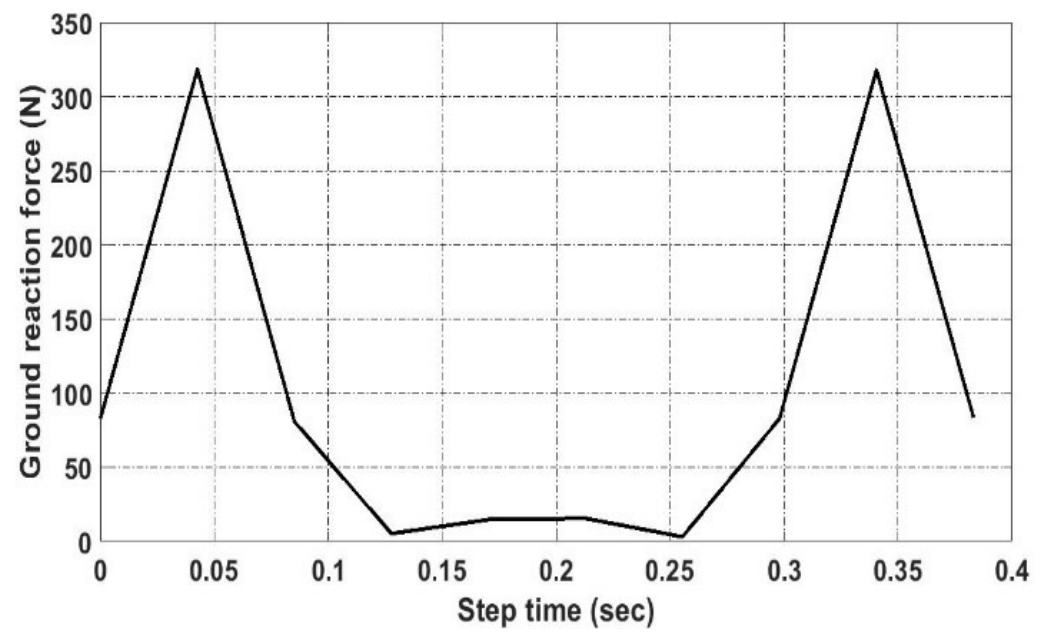

Figure 2. Ground Reaction Force (GRF) of walking

In walking, the torso movement in sagittal plane exhibited one full oscillation from backward to forward position, or the other way around, during a stance phase as shown in Fig. 3. At touch down of one foot, the torso moves in the direction of the same side and reached an extreme position during the period of a single support. Experimentally, this pattern of oscillation of torso angle is found to be approximately asymmetric [18]. Since the motion of the torso during walking is found to be very small, the oscillations are difficult to observe because of its small amplitudes [18].

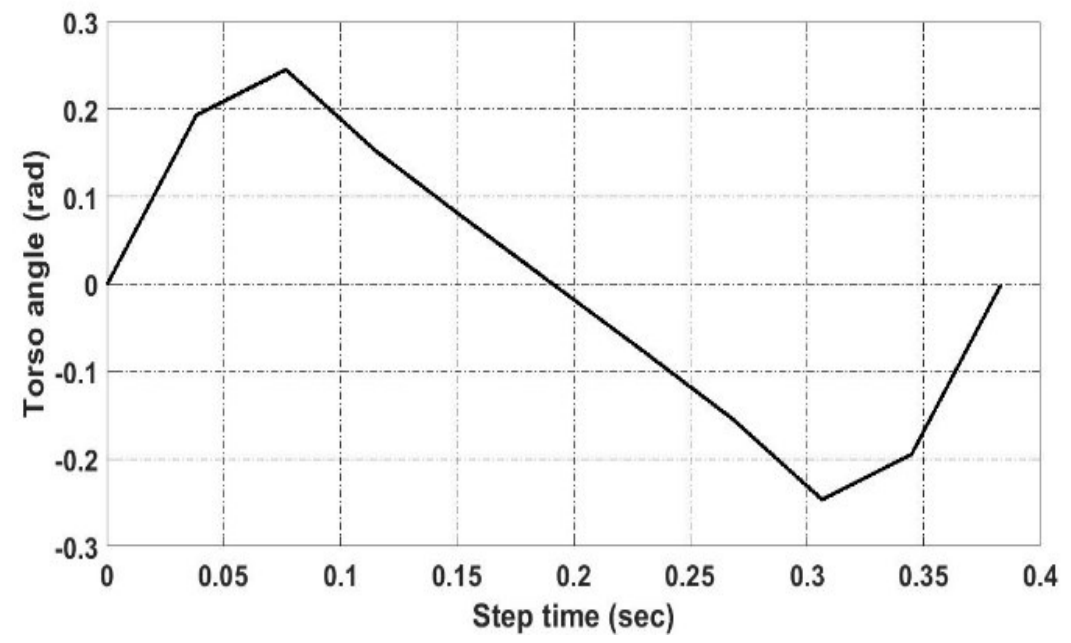

Figure 3. Torso angle during walking

Fig. 4 shows that as the step size increases, the angular displacement of the torso for the biped, peak-to-peak, also increases. For human as reported in [19], this increment in step size causes an 
increment in the range of motion of body segments and joints, which include the torso as well.

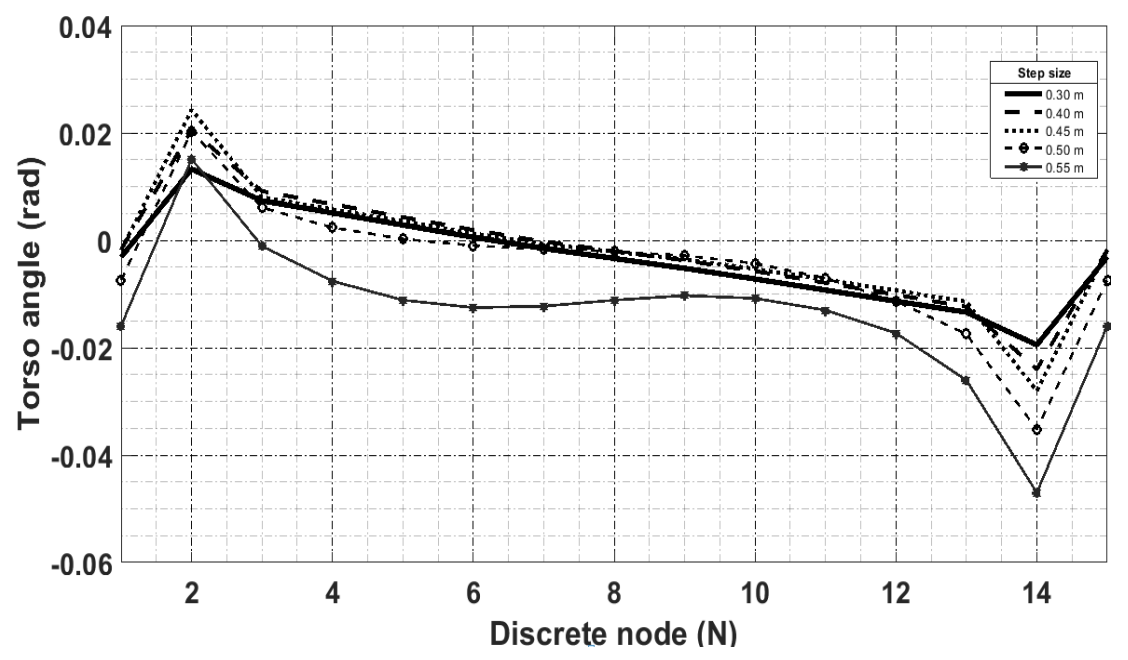

Figure 4. Oscillation of torso at different walking step sizes

Moreover, as the speed of walking increases, the amount of torso angular displacement decreases as shown in Fig. 5. This phenomenon is mainly attributed due to the walking speed, as it increases; it leads to an increase in the range of motion of the lower extremity joints to decrease the vertical shift in the body center of gravity, which is located in the torso [20]. The body behaves in this way in order to minimize the cost of transport by minimizing the movement of the center of gravity [15].

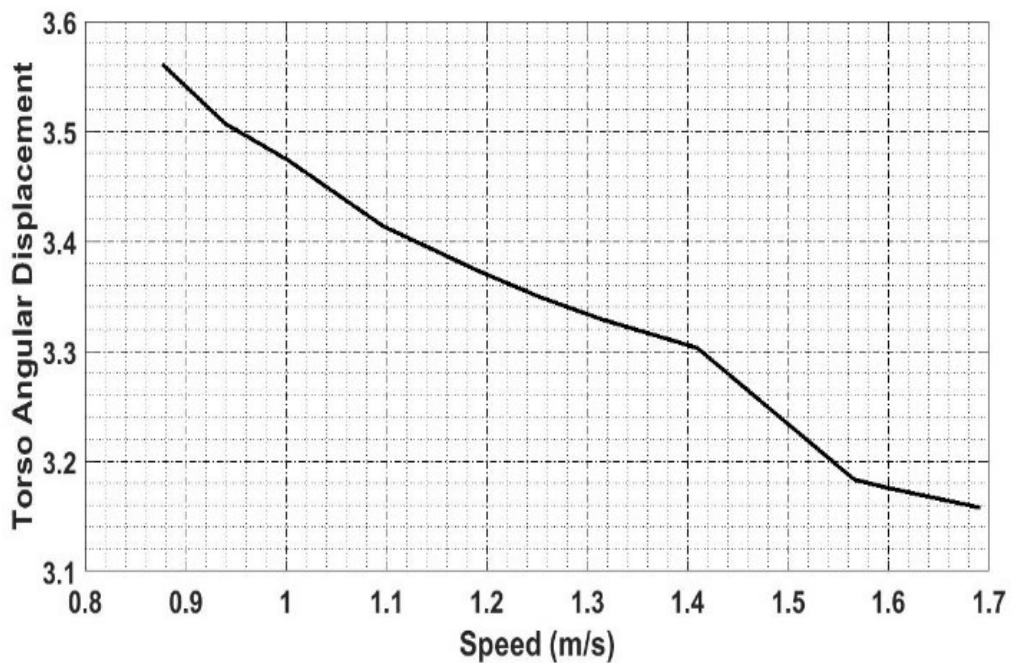

Figure 5. Angular Displacement of torso at different walking speed

\subsection{Running}

In legged locomotion, for a human to move with higher speed they must contract their muscles faster in order to move their limbs more quickly and reduce the amount of time that the feet are in contact with the ground. This process requires more metabolic energy. The optimal gait for high values of speed is impulsive run gait. In this gait, the biped express a high value and impulse of ground reaction force that represents the small stance phase with the start of the step. After this high impulse force, the profile of ground reaction force shows a zero force until the end of the step that represents the flight phase. This high impulse simulates the fast and strong interaction of the foot with the ground. Fig. 6 shows the GRF for impulsive run gait for the case of speed $\mathrm{V}=7 \mathrm{~m} / \mathrm{s}$ and step length $\mathrm{D}=0.2 \mathrm{~m}$. 

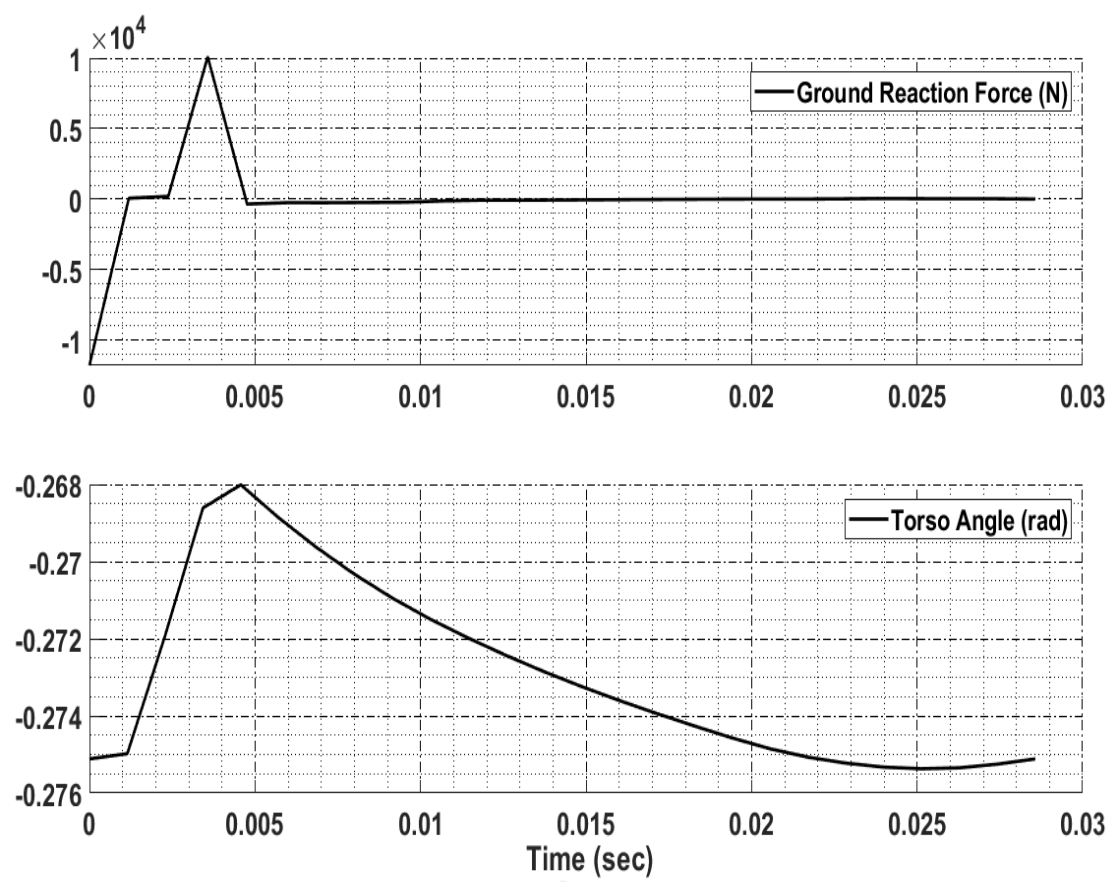

Figure 6. Ground reaction force and torso angle at high running speed $(7 \mathrm{~m} / \mathrm{s})$

Between every two impulsive stance phases, there is a flight phase. In this flight phase, the two legs are off the ground and the body flies in the air, so the ground reaction force is zero in this case [21]. During impulsive run, the body performs negative work to reduce the reaction force to zero in the flight phase and then performs positive work to increase the reaction force again in the next stance phase. In running with low speed, the foot contact with the ground is observed when the torso is almost at its most forward position. However, in running with high speed (e.g., $7 \mathrm{~m} / \mathrm{s}$ ), the touchdown of the foot occurred when the torso is in the peak of the backward position and it is just started to lean forward [18] as shown in Fig. 6.
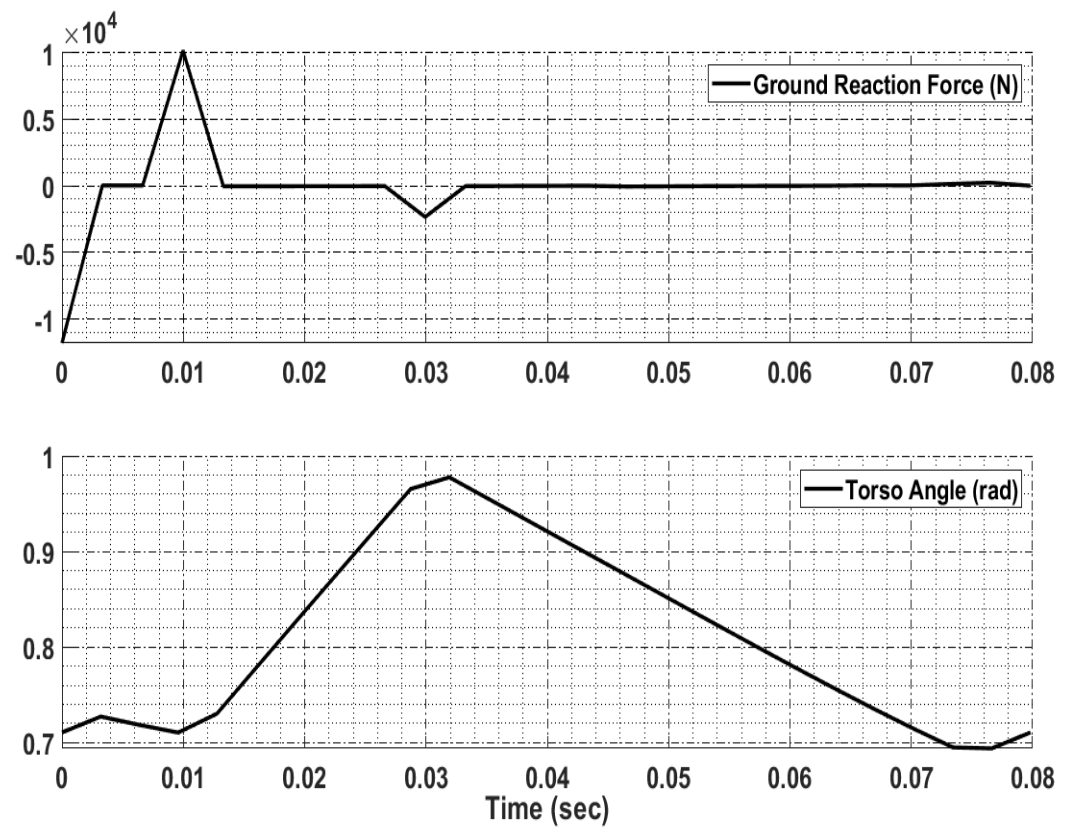

Figure 7. Ground reaction force and torso angle at low running speed $(2.5 \mathrm{~m} / \mathrm{s})$

Fig. 8 shows a reduction in the angular displacement of the torso as the speed increases for the 
aim of minimizing the cost of transport by minimizing the movement of the center of gravity.

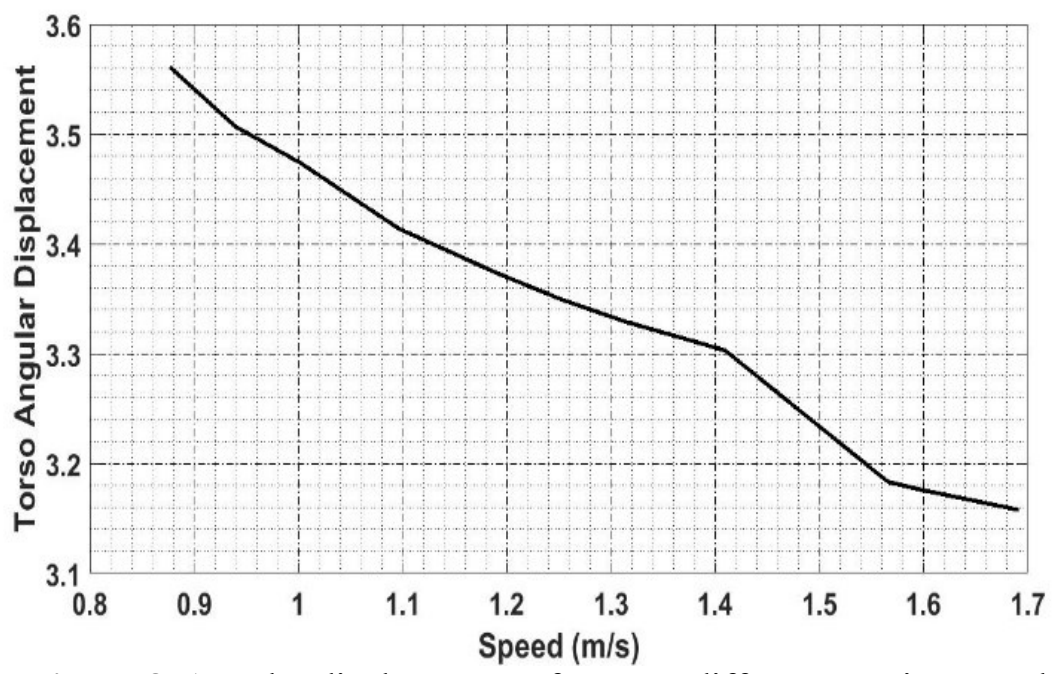

Figure 8. Angular displacement of torso at different running speed

\section{CONCLUSIONS}

Although the proposed model is simple in terms of the involved joints, however, both gait optimization and the biped were able to simulate various features of the human locomotion. The model was able to show the normal profile of the ground reaction force for both running and walking including the oscillation of the torso during both types of gaits. Moreover, the simulation results show that the movement of torso changes with changing the step size and walking speed. Increasing speed of either walking or running tends to reduce the total movement of the torso in the sagittal plane. Studies have shown that this reduction is a result of optimizing the energy required to walk or run. The simulation results showed that the initial position of the torso in running differs as the speed change and the trajectory of the torso changes.

\section{ACKNOWLEDGMENTS}

The authors would like to thank Sultan Qaboos University for their financial support of this project.

\section{REFERENCES}

[1] Morita S, Fujii H, Kobiki T, Minami S, Ohtsuka T. Gait generation method for a compass type walking machine using dynamical symmetry, 2004 IEEE/RSJ International Conference on Intelligent Robots and Systems (IROS)(IEEE Cat. No. 04CH37566) 2004 Oct (Vol. 3, pp. 2825-2830). IEEE.

[2] Srinivasan, M., 2006. Why Walk and Run: Energetic Costs and Energetic Optimality in Simple Mechanics-Based Models of a Bipedal Animal, New York: Cornell University.

[3] Kai T, Shintani T. A discrete mechanics approach to gait generation on periodically unlevel grounds for the compass-type biped robot. International Journal of Advanced Research in Artificial Intelligence. 2013 Sep;2(9):43-51.

[4] Sun, Z., Tian, Y., Li, H. and Wang, J., 2016. A superlinear convergence feasible sequential quadratic programming algorithm for bipedal dynamic walking robot via discrete mechanics and optimal control. Optimal Control Applications and Methods, 37(6), pp.1139-1161.

[5] Han, S.P., 1977. A globally convergent method for nonlinear programming. Journal of optimization theory and applications, 22(3), pp.297-309.

[6] Izmailov, A.F. and Solodov, M.V., 2010. A truncated SQP method based on inexact interior-point solutions of subproblems. SIAM Journal on Optimization, 20(5), pp.2584-2613.

[7] Abdel-Malek, K. \& Arora, J. S., 2013. Human Motion Simulation Predictive Dynamics.

[8] Marrero, J.C., de Diego, D.M. and Martínez, E., 2006. Discrete Lagrangian and Hamiltonian mechanics on Lie groupoids. Nonlinearity, 19(6), p.1313. 
[9] West, M., 2004. Variational integrators (Doctoral dissertation, California Institute of Technology).

[10]Sharma, B.L., 2013. A Glimpse of Discrete Mechanics.

[11]Lew, A., Marsden, J.E., Ortiz, M. and West, M., 2004. An overview of variational integrators.

[12]Kraus, M. (2013). "Variational integrators in plasma physics." arXiv preprint arXiv:1307.5665.

[13]Schultz, J., et al. (2015). Trajectory optimization in discrete mechanics. Differential-Geometric Methods in Computational Multibody System Dynamics, Citeseer.

[14]Perry, J., 1992. Gait Analysis: Normal Pathological Functions. United States of America: SLACK Incorporated.

[15]Farris, D. J. \& Sawicki, G., 2011. The Mechanics and Energetics of Human Walking and Running: a Joint Level Perspective. Journal of The Royal Society Interface, 25 May, pp. 110-118.

[16]AlYahmedi, A. \& Sayari, M., 2014. Efficient Walking Of A Simple Biped With a Torso. Qatar, Middle East Conference on Biomedical Engineering (MECBME), pp. pp. 382-384.

[17]Whittle, M., 2007. Gait analysis, an introduction. USA: Oxford Orthopaedic Engineering Center, ELSEVIER.

[18]Thorstensson AL, Nilsson J, Carlson H, ZOMLEFER MR. Trunk movements in human locomotion. Acta Physiologica Scandinavica. 1984 May;121(1):9-22.

[19]Whitcome, K.K., Miller, E.E. and Burns, J.L., 2017. Pelvic rotation effect on human stride length: Releasing the constraint of obstetric selection. The Anatomical Record, 300(4), pp.752-763.

[20]Dugan, S.A. and Bhat, K.P., 2005. Biomechanics and analysis of running gait. Physical Medicine and Rehabilitation Clinics, 16(3), pp.603-621.

[21]Tongen, A. and Wunderlich, R.E., 2010. Biomechanics of running and walking. Mathematics and Sports, 43, pp.1-12. 